\title{
ON THE SIGN OF A MULTIPLE INTEGRAL
}

\section{J. MORDELL}

(Received 9 April 1962)

One proof of Minkowski's fundamental theorem on lattice points in an n-dimensional parallelopiped depends upon a multiple Fourier expansion. Its terms involve multiple integrals which are easily evaluated and so are shown to have positive values. Then the expansion takes only positive values and the desired proof follows at once. It seems of interest to note a multiple integral which assumes only positive values. We have the

\section{THEOREM}

Suppose that for $x_{1} \geqq 0, x_{2} \geqq 0, \cdots, x_{n} \geqq 0, f(x)=f\left(x_{1}, x_{2}, \cdots, x_{n}\right)$ is defined, that $f(x) \rightarrow 0$ when any variable tends to infinity, and that

$$
(-1)^{n} \frac{\partial^{n} f(\boldsymbol{x})}{\partial x_{1} \cdots \partial x_{n}} \geqq 0 .
$$

Suppose also that

$$
\int \frac{\partial^{r} f(\boldsymbol{x})}{\partial x_{1} \partial x_{2} \cdots \partial x_{r}} d x_{r} \quad(r=1, \cdots, n)
$$

exists for all finite values of the $x$, that the functions $g_{r}(x)$ are continuous for all $x \geqq 0$, and that the

$$
h_{r}(x)=\int_{0}^{x} g_{r}(x) d x, \quad(r=1,2, \cdots, n)
$$

are bounded and non-negative for all $x \geqq 0$.

Then the repeated integral (in the order $x_{1}, x_{2}, \cdots$ )

$$
I_{n}=\int_{0}^{\infty} \int_{0}^{\infty} \cdots \int_{0}^{\infty} f(x) g_{1}\left(x_{1}\right) \cdots g_{n}\left(x_{n}\right) d x_{1} \cdots d x_{n}
$$

converges and $I_{n} \geqq 0$. Also

(4) $I_{n}=(-1)^{n} \int_{0}^{\infty} \int_{0}^{\infty} \cdots \int_{0}^{\infty} \frac{\partial^{n} f(x)}{\partial x_{1} \cdots \partial x_{n}} h_{1}\left(x_{1}\right) \cdots h_{n}\left(x_{n}\right) d x_{1} \cdots d x_{n}$.

The integral (4) converges and its value is independent of the order of integration. For it is majorized by the integral obtained by omitting the $h$ terms. Also the integral 


$$
\int_{L_{1}^{\prime}}^{L_{1}} \cdots \int_{L^{\prime}{ }_{n}}^{L_{n}} \frac{\partial^{n} f(x)}{\partial x_{1} \cdots \partial x_{n}} d x_{1} \cdots d x_{n}
$$

which is easily evaluated in terms of $f(x)$ where the $x$ 's take the value $L_{1}, L_{1}^{\prime}$ etc., (see (6), (7)) is seen to tend to zero independently of the way the $L \rightarrow \infty$. A simple case is when $g_{r}\left(x_{r}\right)=\sin \pi x_{r}$.

We first show that

(5) $\frac{\partial f(x)}{\partial x_{r}} \leqq 0, \quad \frac{\partial^{2} f(x)}{\partial x_{r} \partial x_{s}} \geqq 0, \quad \frac{\partial^{3} f(x)}{\partial x_{r} \partial x_{s} \partial x_{\imath}} \leqq 0$ etc. $\quad\left(\begin{array}{l}r, s, t=1,2, \cdots, n, \\ r \neq s, s \neq t, t \neq r, \text { etc. }\end{array}\right)$

It will suffice to prove the result for $n=3$. We denote the variables by $x, y, z$, and suppose that $x_{2}>x_{1} \geqq 0, y_{2}>y_{1} \geqq 0, z_{2}>z_{1} \geqq 0$. Then from (1),

$$
\int_{x_{1}}^{x_{2}} \int_{y_{1}}^{y_{2}} \int_{z_{1}}^{z_{1}} \frac{\partial^{3} f(x, y, z)}{\partial x \partial y \partial z} d x d y d z \leqq 0
$$

This integral is easily evaluated and gives

$$
\begin{array}{r}
f\left(x_{2}, y_{2}, z_{2}\right)-f\left(x_{2}, y_{1}, z_{2}\right)-f\left(x_{2}, y_{2}, z_{1}\right)+f\left(x_{2}, y_{1}, z_{1}\right) \\
\leqq f\left(x_{1}, y_{2}, z_{2}\right)-f\left(x_{1}, y_{1}, z_{2}\right)-f\left(x_{1}, y_{2}, z_{1}\right)+f\left(x_{1}, y_{1}, z_{1}\right) .
\end{array}
$$

In this, put $y_{2}=\infty, z_{2}=\infty$. Then $f\left(x_{2}, y_{1}, z_{1}\right) \leqq f\left(x_{1}, y_{1}, z_{1}\right)$ and so $f(x, y, z)$ is a decreasing function of $x$ and so $\partial f(x, y, z) / \partial x \leqq 0$. We remark, this implies $f(x, y, z) \geqq 0$ since $f(\infty, y, z)=0$. Put next $z_{2}=\infty$ in (7). Then

$$
f\left(x_{1}, y_{1}, z_{1}\right)-f\left(x_{1}, y_{2}, z_{1}\right)-f\left(x_{2}, y_{1}, z_{1}\right)+f\left(x_{2}, y_{2}, z_{1}\right) \geqq 0 \text {. }
$$

Divide by $y_{1}-y_{2}$ and then make $y_{2} \rightarrow y_{1}$. Then

$$
\frac{\partial f\left(x_{1}, y_{1}, z_{1}\right)}{\partial y_{1}}-\frac{\partial f\left(x_{2}, y_{1}, z_{1}\right)}{\partial y_{1}} \leqq 0 \text {. }
$$

Hence $\partial f(x, y, z) / \partial y$ is an increasing function of $x$ and so $\partial^{2} f(x, y, z) / \partial x \partial y \geqq 0$ etc.

We begin with a well known case when $n=1$ and write

$$
\begin{aligned}
& I_{1}=\int_{0}^{L} f(x) g(x) d x \\
& J_{1}=\int_{0}^{L} \frac{d f(x)}{d x} h(x) d x .
\end{aligned}
$$

Writing $g(x) d x=d(h(x))$, we have from (8), on integrating by parts,

$$
I_{1}=f(L) h(L)-J_{1} \text {. }
$$


When $L \rightarrow \infty, J_{1}$ converges since it is majorized by $\int_{0}^{L}-(d f(x) / d x) d x$ which converges. Since $d f(x) / d x \leqq 0, \int_{0}^{\infty} d f(x) / d x h(x) d x$ converges to a negative value, and hence $\int_{0}^{\infty} f(x) g(x) d x$ converges to a positive value. We take next $n=2$, and write

$$
\begin{aligned}
& I_{2}=\int_{0}^{L} \int_{0}^{M} f(x, y) g_{1}(x) g_{2}(y) d x d y, \\
& J_{2}=\int_{0}^{L} \int_{0}^{M} \frac{\partial^{2} f(x, y)}{\partial x \partial y} h_{1}(x) h_{2}(y) d x d y .
\end{aligned}
$$

In (10), replace $f(x)$ by $f(x, y) g_{2}(y)$ and integrate both sides for $y$ between 0 and $M$. The left hand side becomes $I_{2}$, and so

$$
I_{2}=h_{1}(L) \int_{0}^{M} f(L, y) g_{2}(y) d y-\int_{0}^{L} \int_{0}^{M} \frac{\partial f(x, y)}{\partial x} h_{1}(x) g_{2}(y) d x d y
$$

As in $I_{1}$, the first integral is $h_{2}(M) f(L, M)-\int_{0}^{M}(\partial f(L, y) / \partial y) h_{2}(y) d y$. So for the second integral, integrating first for $y$, we have

$$
h_{2}(M) \frac{\partial f(x, M)}{\partial x}-\int_{0}^{M} \frac{\partial^{2} f(x, y)}{\partial x \partial y} h_{2}(y) d y
$$

Hence we have

$$
\begin{gathered}
I_{2}=h_{1}(L) h_{2}(M) f(L, M)-h_{1}(L) \int_{0}^{M} \frac{\partial f(L, y)}{\partial y} h_{2}(y) d y \\
-h_{2}(M) \int_{0}^{L} \frac{\partial f(x, M)}{\partial x} h_{1}(x) d x+\int_{0}^{L} \int_{0}^{M} \frac{\partial^{2} f(x, y)}{\partial x \partial y} h_{1}(x) h_{2}(y) d x d y
\end{gathered}
$$

Make $L \rightarrow \infty, M \rightarrow \infty$. The two first integrals $\rightarrow 0$ because of their obvious majorizers, and we have the result of the theorem with $n=2$.

We now deal with the case when $n=3$. Here

$$
\begin{aligned}
& I_{3}=\int_{0}^{L} \int_{0}^{M} \int_{0}^{N} f(x, y, z) g_{1}(x) g_{2}(y) g_{3}(z) d x d y d z \\
& J_{3}=\int_{0}^{L} \int_{0}^{M} \int_{0}^{N} f(x, y, z) h_{1}(x) h_{2}(y) h_{3}(z) d x d y d z
\end{aligned}
$$

We deduce the result corresponding to (13) by replacing $f(x, y)$ in (11) and (13) by $g_{3}(z) f(x, y, z)$ and integrating the new $I_{2}$ in (13) for $z$ from 0 to $N$. The result and the proof take such a shape that it is quite clear that the obvious general result follows by induction, and so there is no need to argue from $n$ to $n+1$. We show that 


$$
\begin{aligned}
I_{3}= & h_{1}(L) h_{2}(M) h_{3}(N) f(L, M, N)-\sum h_{2}(M) h_{3}(N) \int_{0}^{L} \frac{\partial f(x, M, N)}{\partial x} h_{1}(x) d x \\
& +\sum h_{1}(L) \int_{0}^{M} \int_{0}^{N} \frac{\partial^{2} f(L, y, z)}{\partial y \partial z} h_{2}(y) h_{3}(z) d y d z \\
& -\int_{0}^{L} \int_{0}^{M} \int_{0}^{N} \frac{\partial^{3} f(x, y, z)}{\partial x \partial y \partial z} h_{1}(x) h_{2}(y) h_{3}(z) d x d y d z .
\end{aligned}
$$

Making $L \rightarrow \infty, M \rightarrow \infty, N \rightarrow \infty$, all the integrals except the last have obvious majorizers which all converge, and tend to zero. Hence we have the case $n=3$ of the theorem.

We take each of the four terms in the new (13) in turn.

Firstly

$$
\int_{0}^{N} f(L, M, z) g_{3}(z) d z=f(L, M, N) h_{3}(N)-\int_{0}^{N} \frac{\partial f(L, M, z)}{\partial z} h_{3}(z) d z .
$$

Secondly

$$
\int_{0}^{M} \int_{0}^{N} \frac{\partial f(L, y, z)}{\partial y} h_{2}(y) g_{3}(z) d y d z
$$

on integrating by parts for $z$ becomes

$$
h_{3}(N) \int_{0}^{M} \frac{\partial f(L, y, N)}{\partial y} h_{2}(y) d y-\int_{0}^{M} \int_{0}^{N} \frac{\partial^{2} f(L, y, z)}{\partial y \partial z} h_{2}(y) h_{3}(z) d y d z .
$$

The third integral is similarly

$$
h_{3}(N) \int_{0}^{L} \frac{\partial f(x, M, N)}{\partial x} h_{1}(x) d x-\int_{0}^{L} \int_{0}^{N} \frac{\partial^{2} f(x, M, z)}{\partial x \partial z} h_{1}(x) h_{3}(z) d x d z,
$$

The fourth integral, on noting that

$$
\int_{0}^{N} \frac{\partial^{2} f(x, y, z)}{\partial x \partial y} g_{3}(z) d z=h_{3}(N) \frac{\partial^{2} f(x, y, N)}{\partial x \partial y}-\int_{0}^{N} \frac{\partial^{3} f(x, y, z)}{\partial x \partial y \partial z} h_{3}(z) d z
$$

becomes

$$
\begin{aligned}
h_{3}(N) \int_{0}^{L} \int_{0}^{M} \frac{\partial^{2} f(x, y, N)}{\partial x \partial y} h_{1}(x) h_{2}(y) d x d y \\
-\int_{0}^{L} \int_{0}^{M} \int_{0}^{N} \frac{\partial^{3} f(x, y, z)}{\partial x \partial y \partial z} h_{1}(x) h_{2}(y) h_{3}(z) d x d y d z .
\end{aligned}
$$

Gathering the terms together, we have (16).

We conclude by noting a simple result when the range of integration is finite. We now consider 


$$
I_{n}^{\prime}=\iint \cdots \int f(x) g_{1}\left(x_{1}\right) g_{2}\left(x_{2}\right) g_{n}\left(x_{n}\right) d x_{1} \cdots d x_{n},
$$

where the integration is taken over the bounded region

$$
x_{1} \geqq 0, \cdots x_{n} \geqq 0, g(x) \leqq 0,
$$

where $g(x)$ is such that it gives $x_{1} \leqq h_{1}\left(x_{2}, \cdots, x_{n}\right)$, where $h_{1}\left(x_{2}, \cdots, x_{n}\right)$ is a continuous function of the variables $x_{2}, \cdots, x_{n}$ for $x_{2} \geqq 0, \cdots, x_{n} \geqq 0_{2}$ and similarly for $x_{2}, \cdots, x_{n}$; and that the $g_{r}(x), h_{r}(x)$ are as before. Suppose that if $g(x)=0$, then

$$
\frac{\partial f(x)}{\partial x_{1}}, \frac{\partial^{2} f(x)}{\partial x_{1} \partial x_{2}}, \cdots, \frac{\partial^{n-1} f(x)}{\partial x_{1} \cdots / x_{n-1}},
$$

are all zero. We suppose these differential coefficients are integrable in turn for $x_{1}, x_{2}, \cdots, x_{n-1}$ respectively. Then if $(-1)^{n}\left(\partial^{n} f(x) / \partial x_{1} \cdots \partial x_{n}\right) \geqq 0$,

$$
I_{n}^{\prime}=\iint \cdots \int \frac{\partial^{n} f(x)}{\partial x_{1} \cdots \partial x_{n}} h_{1}\left(x_{1}\right) \cdots h_{n}\left(x_{n}\right) d x_{1} \cdots d x_{n}
$$

taken over (18).

The proof is obvious on integrating by parts for $x_{1}, x_{2}, \cdots, x_{n}$ successively in (17). The result shows that $I_{n}^{\prime} \geqq 0$.

This work has been supported in part by the National Science Foundation, Washington, D.C.

University of Arizona, Tucson, Arizona, U.S.A.

St. John's College, Cambridge, England 